\title{
Prenatal risk factors for Tourette Syndrome: a systematic review
}

Ting-Kuang $\mathrm{Chao}^{1}$, Jing $\mathrm{Hu}^{2}$ and Tamara Pringsheim ${ }^{2,3^{*}}$

\begin{abstract}
Background: Tourette Syndrome (TS) appears to be an inherited disorder, although genetic abnormalities have been identified in less than 1\% of patients, and the mode of inheritance is uncertain. Many studies have investigated environmental factors that might contribute to the onset and severity of tics and associated comorbidities such as obsessive compulsive disorder (OCD) and attention deficit hyperactive disorder (ADHD). A systematic review and qualitative analysis were performed to provide a broad view of the association between pre- and perinatal factors and TS.

Methods: The Medline, Embase and PsycINFO databases were searched using terms specific to Tourette's syndrome and keywords such as "pregnancy", "prenatal", "perinatal", "birth" and "neonatal". Studies were limited to studies on human subjects published in English or French through October 2012.

Results: 22 studies were included. Studies were of limited methodological quality, with most samples derived from specialty clinics, and most exposures ascertained retrospectively. The majority of the results for demographic factors of parents, including age, education, socioeconomic status, and marital status, revealed no significant association with the onset of TS, or the presence of comorbidity. Many factors were reported to be significantly associated with the onset of TS, the presence of comorbidity and symptom severity, but the most consistently reported factors were maternal smoking and low birth weight.

Conclusions: There are few studies evaluating the relationship between pre and perinatal events and TS, and existing studies have major limitations, including the use of clinic rather than epidemiologically derived samples, retrospective data collection on pre and perinatal events and multiple hypothesis testing without appropriate statistical correction. The mechanism by which prenatal and perinatal adversities could lead to TS onset or symptom severity is unknown, but may be related to changes in the dopaminergic system as a result of early brain injury.
\end{abstract}

Keywords: Tourette syndrome, Tic disorders, Prenatal morbidity, Risk factors

\section{Background}

Tourette Syndrome (TS) appears to be an inherited disorder, although genetic abnormalities have been identified in less than $1 \%$ of patients, and the exact mode of inheritance has not been determined [1]. Some genetic studies have reported infrequent associations of specific genes with TS, including the mutation of SLITRK1 encoding SLIT and NTRK-like protein 1 and a rare functional

\footnotetext{
* Correspondence: tmprings@ucalgary.ca

${ }^{2}$ Department of Clinical Neurosciences, University of Calgary, Calgary, Canada

${ }^{3}$ Alberta Children's Hospital, C4-431, 2888 Shaganappi Trail NW, Calgary, AB

T3B 6A8, Canada

Full list of author information is available at the end of the article
}

mutation in the HDC gene encoding L-histidine decarboxylase [2-9]. While current research suggests that genetic factors confer the greatest risk for the development of TS, many studies have investigated environmental factors that might contribute to the onset of TS, associated comorbidities and more severe symptoms. Among these environmental factors, many studies report that pre- and perinatal factors may play an important role in the pathogenesis of TS [10-28]. However, analysis of these studies has revealed inconsistent results.

We performed a systematic review to gain a complete perspective on the association between pre- and peri-natal adversities and TS, including the onset of symptoms, symptom

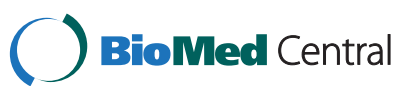


severity, and the presence and severity of comorbidities. There are no up to date systematic reviews focusing on this issue. In this article, a systematic review and qualitative analysis were performed to provide a broad view of the association between pre- and perinatal factors and TS.

\section{Methods}

\section{Electronic searches and selection of studies}

The Medline, Embase and PsycINFO databases were searched using terms specific to Tourette's syndrome and keywords such as "pregnancy", "prenatal", "perinatal", "birth" and "neonatal" (Additional file 1). Studies were limited to studies on human subjects published in English or French through October 2012. Two reviewers independently screened the titles and abstracts. Review articles, commentary articles, editorials, animal studies, and case-reports were excluded. Potentially relevant articles for full-text review were identified by reading abstracts and titles. Full text review was performed on any study for which the abstract or title suggested that the study evaluated pre or perinatal risk factors associated with the development of TS or related to TS severity. Disagreements were resolved by consensus between the two reviewers. Full texts of the selected articles were further reviewed and the following criteria were used to include articles for data extraction: 1) studies specific factors in the prenatal and perinatal period 2) evaluates association with the diagnosis or symptom severity of Tourette syndrome or its comorbidities 3) original study with fully published article available.

\section{Data extraction}

The following information was extracted onto piloted forms: 1) First author, year and geographic location of the study 2) case definition and ascertainment, sources and numbers of cases and controls, matching factors 3) severity scales for Tourette's syndrome or its comorbidities 4) specific risk factors 5) study results, including risk estimate and indicators of statistical significance.

\section{Results}

The electronic searches yielded 141 abstracts, with 31 abstracts selected for full-text review [10-40]. After full text review of these 31 articles, 9 were excluded because only case series were studied [31,32,35,37,38], only poster abstract was available [33], or the contents didn't include pre- or peri-natal adversities $[10,34,40]$. Because of the extreme diversities among studies, the included 22 articles were further categorized according to the different purpose, study design, investigated factors, and outcome. In these 22 articles, two of them were duplicates of data $[11,14]$. Therefore, the data in one report was selected according to the completeness of data contained in both reports [11] (see Figure 1).
Of the 21 studies reporting original data, three were prospective cohort studies, nine were case control studies, four were single group cross sectional studies evaluating disease severity, one was a cross-sectional study with matched case-control pairs, three were twin or family studies, and one was a multiple case group comparative study. The three prospective cohort studies collected pre- and perinatal data prospectively. Two case control studies used pre- and perinatal data recorded on registered birth certificates at the time of delivery, and one cross-sectional study examined children for minor physical anomalies. The remaining fifteen studies collected pre- and perinatal risk factor data retrospectively through parental interview. With the exception of the three prospective cohort studies and one case control study, all samples of cases were derived from speciality clinic settings. See Table 1 for information on study design, sources of cases and controls, data collection and risk factors studied.

\section{Studies investigating the association between pre- and perinatal factors and the onset of Tourette syndrome, and/or risk of comorbidity}

There were 14 articles investigating individual pre- or perinatal factors associated with the onset of TS, risk for comorbidities such as ADHD or OCD, or different genders in TS [11-23]. The summarized results are shown in Table 2. Because of diverse methods for measurement of the risk factors among studies, the results are presented according to the original definition by each study. Similar factors were grouped together but quantitative summarization was not possible because of small sample sizes and the diversity of studies.

\section{Demographic factors and pre-conception health status}

In Table 2, the majority of the results for demographic factors of parents, including age, education, socioeconomic status, and marital status, revealed no significant association with the onset of TS, or the presence of TS comorbidity such as ADHD $[11,13,14,17,20]$. There was only one small study ( 25 cases and 25 controls) in which the mother's age in the group with TS was significantly younger than the control group $(20 \pm 1.4$ versus $23 \pm 3.4$, $\mathrm{p}<0.01)$ [13]. For pre-conception health status, major health problems prior to pregnancy were not associated with the onset of TS, or TS comorbidity, according to the results of two published studies [15,17].

\section{Prenatal period}

During the prenatal period, many studies found that some prenatal adversities were significantly associated with the onset of TS or comorbidities, although the results were not consistent (Table 2). Burd (1999) reported that TS was associated with earlier prenatal care and 


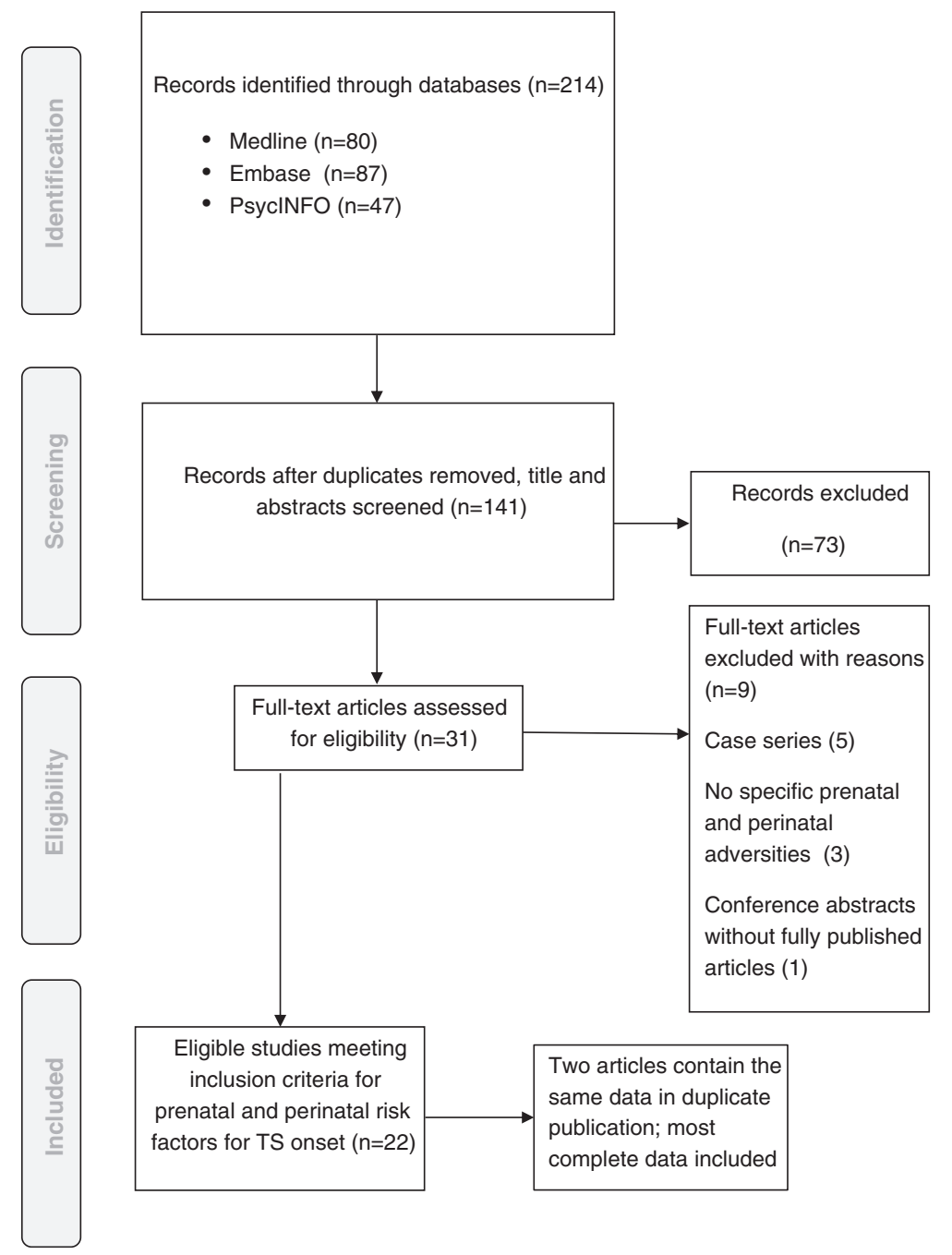

Figure 1 PRISMA flow diagram of included studies.

more prenatal visits compared to the control group [11]. One cross sectional study evaluating the association between minor physical anomalies and TS suggested that early insults during pregnancy might be associated with the onset of TS, as children with TS had a significantly higher number of minor physical anomalies [12]. In 4 studies investigating the association between pregnancy complications and TS onset, the presence of comorbidity, or gender, one study showed a borderline association of one or more pregnancy complications with TS onset $(\mathrm{OR}=2.33,95 \% \mathrm{CI}=0.92-5.89, \mathrm{p}=0.069)$ [18]. Another study demonstrated that one or more pregnancy complications were significantly associated with TS with comorbid ADHD compared to controls, but not associated with TS only patients [17]. One study found that the overall number of prenatal problems was associated with a higher risk of ADHD comorbidity $(\mathrm{OR}=2.97,95 \% \mathrm{CI}$ : $1.27-6.94, \mathrm{p}=0.02)$ [26].
Some individual and combined factors during pregnancy were also investigated, including maternal hypertension, psychosocial stress, antibiotic exposure, or medications/ procedures $[17,19,21]$. In these studies, psychosocial stress was not significantly associated with the onset of TS or TS with ADHD compared to controls. In another study, antibiotic exposure was significantly associated with both TS and TS with ADHD ( $\mathrm{p}=0.02)$ [17].

With regard to maternal smoking, one study revealed borderline results in the association with onset of TS or TS with ADHD compared to the control group. The odds ratios were very high: TS $(\mathrm{OR}=4.6)$, TS with ADHD (OR = 8.5) (Table 2) [17]. The study found that TS with ADHD had an even higher association with maternal smoking than TS only. This trend was also noted in another study- that compared to TS only patients, the presence of ADHD comorbidity was significantly associated with maternal smoking during the 
Table 1 Characteristics of included studies

\begin{tabular}{|c|c|c|c|c|c|c|c|c|c|c|}
\hline Study & Study design & Cases & Controls & Source of cases & $\begin{array}{l}\text { Sources of } \\
\text { controls }\end{array}$ & $\begin{array}{l}\text { Matching } \\
\text { factors }\end{array}$ & $\begin{array}{l}\text { Diagnosis } \\
\text { of TS }\end{array}$ & Data source & $\begin{array}{l}\text { Prospective or } \\
\text { retrospective } \\
\text { collection of } \\
\text { data on } \\
\text { perinatal events }\end{array}$ & Risk factors evaluated \\
\hline $\begin{array}{l}\text { Atladottir } \\
2007 \text { [29] }\end{array}$ & Cohort study & TS & Non-TS & $\begin{array}{l}\text { Danish Medical } \\
\text { Birth Registry } \\
\text { cohort }\end{array}$ & $\begin{array}{l}\text { Danish Medical } \\
\text { Birth Registry } \\
\text { cohort }\end{array}$ & & $\begin{array}{l}\text { ICD-10 in } \\
\text { Psychiatric } \\
\text { Registry }\end{array}$ & Registry & Prospective & Season of birth \\
\hline $\begin{array}{l}\text { Atladottir } \\
2007 \text { [29] }\end{array}$ & Cohort study & TS & Non-TS & $\begin{array}{l}\text { Danish Medical } \\
\text { Birth Registry } \\
\text { cohort }\end{array}$ & $\begin{array}{l}\text { Danish Medical } \\
\text { Birth Registry } \\
\text { cohort }\end{array}$ & & $\begin{array}{l}\text { ICD-10 in } \\
\text { Psychiatric } \\
\text { Registry }\end{array}$ & Registry & Prospective & $\begin{array}{l}\text { Birth year (1990-1991, } \\
\text { 1992-1993, 1994-1995) }\end{array}$ \\
\hline $\begin{array}{l}\text { Bos-Veneman } \\
2010 \text { [27] }\end{array}$ & $\begin{array}{l}\text { Single group } \\
\text { cross-sectional; } \\
\text { comparison of tic } \\
\text { and ADHD severity } \\
\text { in those with/ } \\
\text { without risk factors }\end{array}$ & $\begin{array}{l}\text { TS (62), Chronic } \\
\text { motor (12) or } \\
\text { vocal (1) tics }\end{array}$ & - & $\begin{array}{l}\text { Psychiatry clinic } \\
\text { (60) or TS } \\
\text { Association (15) }\end{array}$ & - & & DSM-IV-TR & $\begin{array}{l}\text { Parent interview } \\
\text { and questionnaire }\end{array}$ & Retrospective & $\begin{array}{l}\text { Pregnancy complications, } \\
\text { Delivery complications, } \\
\text { First-week postnatal } \\
\text { complications, Prenatal } \\
\text { smoking exposure, Prenatal } \\
\text { alcohol exposure }\end{array}$ \\
\hline $\begin{array}{l}\text { Bos-Veneman } \\
2010 \text { [27] }\end{array}$ & $\begin{array}{l}\text { Single group cross- } \\
\text { sectional; comparison } \\
\text { of symptom severity } \\
\text { in those with/ } \\
\text { without risk factors } \\
\text { and genetic factors }\end{array}$ & Tic disorders & - & $\begin{array}{l}\text { Psychiatry clinic } \\
\text { (86) or TS } \\
\text { Association (24) }\end{array}$ & - & & DSM-IV-TR & $\begin{array}{l}\text { Parent interview } \\
\text { and questionnaire }\end{array}$ & Retrospective & $\begin{array}{l}\text { (1) Genetic factors } \\
\text { (Dopamine receptor D4 } \\
\text { (DRD4) and interaction } \\
\text { with environmental } \\
\text { factors; (2) Pregnancy } \\
\text { complications: HT, DM, } \\
\text { infections, (pre)eclampsia, } \\
\text { psychosocial stress; (3) } \\
\text { Delivery complications: } \\
\text { meconium-stained } \\
\text { amniotic fluid, premature } \\
\text { rupture of the membranes, } \\
\text { nuchal cord, fetal } \\
\text { bradycardia, placenta } \\
\text { previa, artificial delivery; } \\
\text { (4) Smoking during } \\
\text { pregnancy }\end{array}$ \\
\hline $\begin{array}{l}\text { Burd } 1999 \\
\text { [11] }\end{array}$ & $\begin{array}{l}\text { Matched Case } \\
\text { control (1:5) }\end{array}$ & TS & Healthy & $\begin{array}{l}\text { North Dakota TS } \\
\text { registry }\end{array}$ & $\begin{array}{l}\text { North Dakota } \\
\text { Health } \\
\text { Department } \\
\text { Registry }\end{array}$ & $\begin{array}{l}\text { Sex, year } \\
\text { of birth, } \\
\text { month of } \\
\text { birth }\end{array}$ & $\begin{array}{l}\text { DSM-III, } \\
\text { DSM-III-R, } \\
\text { DSM-IV }\end{array}$ & Birth certificate data & Prospective & $\begin{array}{l}\text { Birth weight, Apgar } \\
\text { scores, mother's and } \\
\text { father's age and } \\
\text { education, month } \\
\text { prenatal care began, } \\
\text { number of prenatal visits, } \\
\text { gestational age }\end{array}$ \\
\hline $\begin{array}{l}\text { Csabi } 2008 \\
{[12]}\end{array}$ & $\begin{array}{l}\text { Cross-sectional } \\
\text { study with matched } \\
\text { case control pairs }(1: 1)\end{array}$ & TS & Healthy & Outpatient clinic & $\begin{array}{l}\text { Local } \\
\text { elementary } \\
\text { schools }\end{array}$ & $\begin{array}{l}\text { Sex, age, } \\
\text { ethnical } \\
\text { origin }\end{array}$ & DSM-IV & $\begin{array}{l}\text { Mehes Scale for } \\
\text { evaluation of minor } \\
\text { physical anomalies }\end{array}$ & Prospective & $\begin{array}{l}\text { Presence of minor } \\
\text { physical anomalies on } \\
\text { examination }\end{array}$ \\
\hline
\end{tabular}


Table 1 Characteristics of included studies (Continued)

\begin{tabular}{|c|c|c|c|c|c|c|c|c|c|c|}
\hline $\begin{array}{l}\text { Hyde } 1992 \\
{[25]}\end{array}$ & $\begin{array}{l}\text { Twin study; evaluated } \\
\text { differences between } \\
\text { twins in presence of } \\
\text { TS and tic severity }\end{array}$ & TS & - & $\begin{array}{l}\text { Tourette } \\
\text { Syndrome } \\
\text { Association }\end{array}$ & - & Twins & $\begin{array}{l}\text { Diagnostic } \\
\text { interview }\end{array}$ & $\begin{array}{l}\text { Mother completed } \\
\text { questionnaire; } \\
\text { confirmation of data } \\
\text { through review of } \\
\text { medical records } \\
\text { where possible (not } \\
\text { stated how many } \\
\text { this was possible } \\
\text { for) }\end{array}$ & Retrospective & $\begin{array}{l}\text { Birth weight ( } \mathrm{n}=13 \\
\text { pairs), birth order } \\
(\mathrm{n}=8 \text { pairs), handedness } \\
\text { ( } \mathrm{n}=7 \text { pairs), medical } \\
\text { events and environmental } \\
\text { factors ( } \mathrm{n}=13 \text { pairs) }\end{array}$ \\
\hline $\begin{array}{l}\text { Kano } 2002 \\
{[36]}\end{array}$ & $\begin{array}{l}\text { Family study; } \\
\text { probands and first } \\
\text { degree relatives }\end{array}$ & TS & & Not stated & $\begin{array}{l}\text { First degree } \\
\text { relatives }\end{array}$ & & DSM-III & Report from mother & Retrospective & $\begin{array}{l}\text { Premature delivery, use of } \\
\text { forceps, apnea at birth, } \\
\text { cyanosis at birth, slowed } \\
\text { heartbeat }\end{array}$ \\
\hline $\begin{array}{l}\text { Khalifa } 2005 \\
{[13]}\end{array}$ & $\begin{array}{l}\text { Matched Case } \\
\text { control }(1: 1)\end{array}$ & TS & Healthy & $\begin{array}{l}\text { Total population } \\
\text { of children in a } \\
\text { town in central } \\
\text { Sweden }\end{array}$ & $\begin{array}{l}\text { Total population } \\
\text { of children in a } \\
\text { town in central } \\
\text { Sweden }\end{array}$ & $\begin{array}{l}\text { Sex, age, } \\
\text { school }\end{array}$ & DSM-IV & $\begin{array}{l}\text { Interview with } \\
\text { parents and medical } \\
\text { record review }\end{array}$ & Retrospective & $\begin{array}{l}\text { reduced optimality in the } \\
\text { pre-, peri-, and neonatal } \\
\text { periods (modified } \\
\text { Prechtl's non-optimal } \\
\text { score) }\end{array}$ \\
\hline $\begin{array}{l}\text { Klug } 2003[14] \\
\text { (duplicate } \\
\text { data as Burd } \\
\text { 1999) [11] }\end{array}$ & $\begin{array}{l}\text { Matched Case } \\
\text { control (1:5) }\end{array}$ & TS & Healthy & $\begin{array}{l}\text { North Dakota TS } \\
\text { registry }\end{array}$ & $\begin{array}{l}\text { North Dakota } \\
\text { Health } \\
\text { Department } \\
\text { Registry }\end{array}$ & $\begin{array}{l}\text { Sex, year } \\
\text { of birth, } \\
\text { month of } \\
\text { birth }\end{array}$ & Not stated & Birth certificate data & Prospective & $\begin{array}{l}\text { Birth weight, Apgar } \\
\text { scores, mother's and } \\
\text { father's age and } \\
\text { education, month } \\
\text { prenatal care began, } \\
\text { number of prenatal visits, } \\
\text { gestational age, number } \\
\text { of child malformations }\end{array}$ \\
\hline $\begin{array}{l}\text { Kondo } 1992 \\
{[15]}\end{array}$ & Case control & TS & $\begin{array}{l}\text { Duchenne } \\
\text { dystrophy }\end{array}$ & $\begin{array}{l}\text { Neurology } \\
\text { Outpatient Clinic }\end{array}$ & Other hospitals & $\begin{array}{l}\text { None } \\
\text { stated }\end{array}$ & Not stated & $\begin{array}{l}\text { Patient and mother } \\
\text { interview }\end{array}$ & Retrospective & $\begin{array}{l}\text { Prenatal, perinatal and } \\
\text { developmental history }\end{array}$ \\
\hline $\begin{array}{l}\text { Leckman } \\
1990[24]\end{array}$ & $\begin{array}{l}\text { Single group cross- } \\
\text { sectional; comparison } \\
\text { of tics severity in those } \\
\text { with/without risk } \\
\text { factors }\end{array}$ & TS & - & $\begin{array}{l}\text { TS Specialty } \\
\text { Clinic }\end{array}$ & - & & DSM-III & $\begin{array}{l}\text { Interview with } \\
\text { mother; } \\
\text { confirmation with } \\
\text { obstetric records in } \\
61 \%\end{array}$ & Retrospective & $\begin{array}{l}\text { 41-item Obstetric } \\
\text { Complications Scale } \\
\text { (OCS), Perinatal } \\
\text { complication scale (PCS), } \\
\text { Level of Stress severity, } \\
\text { Coping Ability Scale, } \\
41 \text {-item Life Stress } \\
\text { 'Scale (LSS) }\end{array}$ \\
\hline $\begin{array}{l}\text { Leckman } \\
1987[16]\end{array}$ & Twin study & $\begin{array}{l}\text { Monozygotic } \\
\text { twins } \\
\text { disconcordant } \\
\text { for TS }\end{array}$ & $\begin{array}{l}\text { Unaffected } \\
\text { twin }\end{array}$ & Not stated & & Twins & Not stated & Parental interview & Retrospective & Birth weight \\
\hline $\begin{array}{l}\text { Mathews } \\
2006[26]\end{array}$ & $\begin{array}{l}\text { Single group cross- } \\
\text { sectional; comparison } \\
\text { of tics severity in those } \\
\text { with/without risk } \\
\text { factors }\end{array}$ & TS & - & $\begin{array}{l}\text { TS specialty } \\
\text { clinics; TS } \\
\text { Association; } \\
\text { Health care } \\
\text { professionals, } \\
\text { media } \\
\text { advertisements, } \\
\text { schools }\end{array}$ & - & & DSM-IV & Parental interview & Retrospective & $\begin{array}{l}\text { Prenatal problems, } \\
\text { perinatal problems, and } \\
\text { in utero medication } \\
\text { exposure (details in the } \\
\text { article) }\end{array}$ \\
\hline
\end{tabular}


Table 1 Characteristics of included studies (Continued)

\begin{tabular}{|c|c|c|c|c|c|c|c|c|c|c|}
\hline $\begin{array}{l}\text { Motlagh } 2010 \\
\text { [17] }\end{array}$ & Case control & TS, TS + ADHD & Healthy & $\begin{array}{l}\text { TS specialty } \\
\text { clinic and TS } \\
\text { Association }\end{array}$ & $\begin{array}{l}\text { Telemarketing } \\
\text { lists }\end{array}$ & $\begin{array}{l}\text { Age, ZIP } \\
\text { codes }\end{array}$ & DSM-IV & Maternal interview & Retrospective & $\begin{array}{l}\text { Prenatal, obstetric, } \\
\text { medical risk factors, life } \\
\text { circumstances during } \\
\text { pregnancy, birth weight, } \\
\text { perinatal adverse events }\end{array}$ \\
\hline $\begin{array}{l}\text { Pasamanick } \\
1956[18]\end{array}$ & $\begin{array}{l}\text { Matched Case } \\
\text { control (1:1) }\end{array}$ & $\begin{array}{l}\text { Children with } \\
\text { tics }\end{array}$ & Healthy & $\begin{array}{l}\text { Child Psychiatry } \\
\text { clinic }\end{array}$ & $\begin{array}{l}\text { Birth register of } \\
\text { the Bureau of } \\
\text { Vital Records of } \\
\text { the Baltimore } \\
\text { City Health } \\
\text { Department }\end{array}$ & $\begin{array}{l}\text { Same } \\
\text { place of } \\
\text { birth, race, } \\
\text { sex, } \\
\text { maternal } \\
\text { age group }\end{array}$ & Not stated & $\begin{array}{l}\text { Hospital medical } \\
\text { record of birth }\end{array}$ & Prospective & $\begin{array}{l}\text { Pregnancy history, length } \\
\text { of labour, complications } \\
\text { of pregnancy and } \\
\text { delivery, birth weight, } \\
\text { condition of child during } \\
\text { neonatal period }\end{array}$ \\
\hline $\begin{array}{l}\text { Saccomani } \\
2005[23]\end{array}$ & Case control & TS, chronic tics & Healthy & Outpatient clinic & $\begin{array}{l}\text { Offspring of } \\
\text { hospital } \\
\text { personnel }\end{array}$ & $\begin{array}{l}\text { None } \\
\text { stated }\end{array}$ & DSM-IV-TR & Parental interview & Retrospective & $\begin{array}{l}\text { Definition of pre- and } \\
\text { perinatal events: threatened } \\
\text { miscarriage, prematurity, } \\
\text { prolonged labour, umbilical } \\
\text { cord around the neck, } \\
\text { forceps delivery, and } \\
\text { neonatal jaundice }\end{array}$ \\
\hline $\begin{array}{l}\text { Santangelo } \\
1994 \text { [19] }\end{array}$ & $\begin{array}{l}\text { Multiple case group } \\
\text { comparison; } \\
\text { comparison of } \\
\text { presence of } \\
\text { comorbid OCD in } \\
\text { those with/without } \\
\text { risk factors }\end{array}$ & $\mathrm{TS}, \mathrm{TS}+\mathrm{OCD}$ & & TS Association & & & DSM-III-R & Maternal interview & Retrospective & $\begin{array}{l}\text { (1) pregnancy } \\
\text { complications, (2) delivery } \\
\text { complications, (3) } \\
\text { Medications/procedures, } \\
\text { and (4) Coffee, cigarettes, } \\
\text { alcohol }\end{array}$ \\
\hline $\begin{array}{l}\text { Shimada } \\
2012 \text { [20] }\end{array}$ & Case Control & TS & $\begin{array}{l}\text { General } \\
\text { population } \\
\text { data }\end{array}$ & $\begin{array}{l}\text { Psychiatry } \\
\text { outpatient clinic }\end{array}$ & $\begin{array}{l}\text { General } \\
\text { population } \\
\text { database }\end{array}$ & & DSM-IV-TR & $\begin{array}{l}\text { Parent interview } \\
\text { and questionnaire }\end{array}$ & Retrospective & $\begin{array}{l}\text { Parental age, education } \\
\text { level of parents, use of } \\
\text { assistive reproduction, } \\
\text { birth weight, gestational } \\
\text { age }\end{array}$ \\
\hline $\begin{array}{l}\text { Pringsheim } \\
2009 \text { [21] }\end{array}$ & Nested case-control & $\mathrm{TS}+\mathrm{ADHD}$ & TS & $\begin{array}{l}\text { TS Specialty } \\
\text { clinic }\end{array}$ & $\begin{array}{l}\text { TS Specialty } \\
\text { clinic }\end{array}$ & & DSM-IV-TR & $\begin{array}{l}\text { Parental interview } \\
\text { and questionnaire }\end{array}$ & Retrospective & $\begin{array}{l}\text { Maternal smoking, } \\
\text { Maternal alcohol } \\
\text { exposure, Low BW, } \\
\text { Premature, small for GA, } \\
\text { Breathing problems at } \\
\text { birth, Maternal HT, } \\
\text { Operative delivery, } \\
\text { prenatal drug use, }\end{array}$ \\
\hline lida 1996 [22] & Case control & $\mathrm{TS}+\mathrm{OCS}$ & TS & $\begin{array}{l}\text { Outpatient } \\
\text { psychiatry clinic }\end{array}$ & $\begin{array}{l}\text { Outpatient } \\
\text { psychiatry clinic }\end{array}$ & & DSM-III-R & Maternal interview & Retrospective & $\begin{array}{l}\text { Perinatal complications } \\
\text { (postnatal jaundice, cord } \\
\text { around neck, breech } \\
\text { birth, premature birth) }\end{array}$ \\
\hline
\end{tabular}


Table 1 Characteristics of included studies (Continued)

Prospective cohort

Low birth

weight

Regional birth

cohort of low

cohort of low

children

DSM-III-R

Psychiatric diagnosis Prospective

assessed at age 6

by structured

This was a prospective

cohort study of low birth

weight babies who were

examined at age 6 for

examined at age 6 for

psychiatric disorders

including TS. The study

examined the relationship

between neonatal cranial

ultrasound abnormalities

in these children and the

diagnosis of psychiatric

disorders at age 6 . 
Table 2 Pre- and peri-natal factors associated with the onset of Tourette's syndrome (TS), and the presence of comorbidity

\begin{tabular}{|c|c|c|c|c|c|c|}
\hline Factors & Results (Cases vs. Controls)* & $P$ value & Case number & $\begin{array}{l}\text { Control } \\
\text { number }\end{array}$ & Note & Reference \\
\hline \multicolumn{7}{|l|}{ Demographic factors } \\
\hline \multirow[t]{4}{*}{ Mother's age } & 25.69 (5.29) vs. 25.12 (5.2) & $p=0.33$ & 92 & 460 & Matched analysis & Burd 1999 [11] \\
\hline & 20 (1.4) vs. 23 (3.4) & $\mathrm{p}<0.01$ & 25 & 25 & & Khalifa 2005 [13] \\
\hline & 29.8 (4.5) vs. 29.6 & & 66 & 1174999 & $\begin{array}{l}\text { Adjusted for birth } \\
\text { year }\end{array}$ & Shimada 2012 [20] \\
\hline & $\begin{array}{l}\text { TS: } 28.9 \text { (4.4), TS + ADHD: } 28.1 \text { (5.3), Control: } \\
28.3(4.1)\end{array}$ & NS & $\begin{array}{l}\text { TS: } 45, \text { TS + } \\
\text { ADHD:60 }\end{array}$ & 65 & & Motlagh 2010 [17] \\
\hline \multirow[t]{3}{*}{ Father's age } & 28.87 (6.25) vs. 27.78 (5.96) & $p=0.11$ & 92 & 460 & Matched analysis & Burd 1999 [11] \\
\hline & 31.9 (5.0) vs. 32.7 & NS & 65 & 1174999 & $\begin{array}{l}\text { Adjusted for birth } \\
\text { year }\end{array}$ & Shimada 2012 [20] \\
\hline & $\begin{array}{l}\text { TS: } 30.9 \text { (5.0), TS + ADHD: } 30.2 \text { (6.2), Control: } \\
30.4 \text { (5.0) }\end{array}$ & NS & $\begin{array}{l}\text { TS: } 45, \text { TS }+ \\
\text { ADHD:60 }\end{array}$ & 65 & & Motlagh 2010 [17] \\
\hline Mother's education & 12.90 (2.11) vs. 12.64 (1.92) & $p=0.24$ & 92 & 460 & Matched analysis & Burd 1999 [11] \\
\hline Father's education & 12.69 (2.44) vs. 12.67 (2.15) & $p=0.96$ & 92 & 460 & Matched analysis & Burd 1999 [11] \\
\hline Parent's education & $\begin{array}{l}\text { TS: } 15.5 \text { (2.0), TS + ADHD: } 14.9 \text { (1.8), Control: } \\
15.3(2.0)\end{array}$ & NS & $\begin{array}{l}\text { TS: } 45, \text { TS }+ \\
\text { ADHD:60 }\end{array}$ & 65 & & Motlagh 2010 [17] \\
\hline Mean socioeconomic status & $\begin{array}{l}\text { TS: } 50.4 \text { (2.7), TS + ADHD: } 47.4 \text { (10.6), Control: } \\
48.5 \text { (10.7) }\end{array}$ & NS & $\begin{array}{l}\text { TS: } 45, \text { TS }+ \\
\text { ADHD:60 }\end{array}$ & 65 & & Motlagh 2010 [17] \\
\hline $\begin{array}{l}\text { Socio-economic status, parental } \\
\text { education or divorce rate }\end{array}$ & No difference (no data provided) & NS & 25 & 25 & & Khalifa 2005 [13] \\
\hline Marital status & $4 / 92$ vs. $36 / 460$ & $p=0.93$ & 92 & 460 & & Klug 2003 [14] \\
\hline \multicolumn{7}{|l|}{ Pre-conception condition } \\
\hline \multirow{2}{*}{$\begin{array}{l}\text { Major diseases before pregnancy } \\
\text { or pre-existing medical conditions }\end{array}$} & (20/42 vs. $21 / 43) \mathrm{OR}=0.95,95 \% \mathrm{Cl}=0.41-2.23$ & $p=0.91$ & 42 & 43 & & Kondo 1982 [15] \\
\hline & $\begin{array}{l}\text { TS vs. Control ( } 1 / 45 \text { vs. } 4 / 65) \text {; TS + ADHD vs. } \\
\text { Control (4/60 vs. } 4 / 65)\end{array}$ & NS & $\begin{array}{l}\text { TS: } 45, \text { TS }+ \\
\text { ADHD:60 }\end{array}$ & 65 & & Motlagh 2010 [17] \\
\hline \multicolumn{7}{|l|}{ Pre-natal period } \\
\hline Month prenatal care began & 2.47 (1.27) vs. 3.01 (1.40) & $p=0.001$ & 92 & 460 & Matched analysis & Burd 1999 [11] \\
\hline Prenatal visits & 10.00 (3.57) vs. 8.51 (3.22) & $\mathrm{p}<0.001$ & 92 & 460 & Matched analysis & Burd 1999 [11] \\
\hline Prenatal care begun after first trimester & $\mathrm{OR}=0.49(0.27-0.90),(14 / 92$ vs. $123 / 460)$ & $p=0.03$ & 92 & 460 & & Burd 1999 [11] \\
\hline Minor physical anomalies (numbers) & $\begin{array}{l}5.46(2.15) \text { vs. } 1.11(1.18) ; \text { Mann-Whitney U-test } \\
49.50,-Z=-4.92\end{array}$ & $p=0.001$ & 24 & 24 & & Csabi 2008 [12] \\
\hline Number of prenatal problems & Presence of $\mathrm{ADHD}, \mathrm{OR}=2.97,95 \% \mathrm{Cl}: 1.27-6.94$ & $p=0.02$ & 180 & & & Mathews 2006 [26] \\
\hline Disordered pregnancies & $(12 / 42$ vs. $15 / 43) \mathrm{OR}=0.75,95 \% \mathrm{Cl}=0.30-1.87$ & $p=0.53$ & 42 & 43 & & Kondo 1982 [15] \\
\hline \multirow[t]{2}{*}{ Any pregnancy complication } & Male TS vs. Female TS: 63\% vs. 83\% & NS & $46 \mathrm{M}$ & $7 F$ & & Santangelo 1994 [19] \\
\hline & TS + OCD vs. TS: $67 \%$ vs. $65 \%$ & NS & 15 & 34 & & Santangelo 1994 [19] \\
\hline
\end{tabular}

\section{Mother's education \\ Father's education}

Mean socioeconomic status

Major diseases before pregnancy

\section{Pre-natal perio}

Prenatal visits

Prenatal care begun after first trimester

Number of prenatal problem

Derdered pregnancies

TS + OCD vs. TS: $67 \%$ vs. $65 \%$ 
Table 2 Pre- and peri-natal factors associated with the onset of Tourette's syndrome (TS), and the presence of comorbidity (Continued)

\begin{tabular}{|c|c|c|c|c|c|c|}
\hline \multirow[t]{3}{*}{ One or more pregnancy complication } & $17 / 51$ vs. $9 / 51(\mathrm{OR}=2.33,95 \% \mathrm{Cl}=0.92-5.89)$ & $p=0.07$ & 51 & 51 & & $\begin{array}{l}\text { Pasamanick } 1956 \\
\text { [18] }\end{array}$ \\
\hline & TS + ADHD: 27/60, Control: 16/65 & $p=0.01$ & TS + ADHD:60 & 65 & & Motlagh 2010 [17] \\
\hline & TS: 17/45, Control: 16/65 & NS & TS: 45 & 65 & & Motlagh 2010 [17] \\
\hline Maternal hypertension & TS + ADHD vs. TS, OR= 1.04 (95\% Cl: 0.44-2.42) & $p=0.93$ & 181 & 172 & Unadjusted OR & Pringsheim 2009 [21] \\
\hline Severe psychosocial stress & $\begin{array}{l}\text { TS vs. Control }(10 / 45 \text { vs. } 5 / 62, \mathrm{OR}=2.6,95 \% \\
\mathrm{Cl}=0.08-8.7, \mathrm{p}=0.11) ; \mathrm{TS}+\mathrm{ADHD} \text { vs. Control } \\
(11 / 55 \text { vs. } 5 / 62, \mathrm{OR}=3.1,95 \% \mathrm{Cl}=0.9-11.1 \\
p=0.07)\end{array}$ & $\begin{array}{l}p=0.11 \text { and } \\
0.07\end{array}$ & $\begin{array}{l}\text { TS: } 45, \text { TS + } \\
\text { ADHD:60 }\end{array}$ & 65 & $\begin{array}{l}\text { Adjusted for } \\
\text { gender }\end{array}$ & Motlagh 2010 [17] \\
\hline Antibiotics exposure in pregnancy & TS: 11/45, TS + ADHD: 11/60, Control: 6/65 & both $\mathbf{p}<0.05$ & $\begin{array}{l}\text { TS: } 45, \text { TS + } \\
\text { ADHD: } 60\end{array}$ & 65 & & Motlagh 2010 [17] \\
\hline \multirow[t]{2}{*}{ Medication/procedures } & Male TS vs. Female TS: $37 \%$ vs. $50 \%$ & NS & $46 \mathrm{M}$ & $7 F$ & & Santangelo 1994 [19] \\
\hline & TS + OCD vs. TS: $27 \%$ vs. $44 \%$ & NS & 15 & 34 & & Santangelo 1994 [19] \\
\hline Number of medications exposed to in utero & Presence of $\mathrm{ADHD}, \mathrm{OR}=0.50,95 \% \mathrm{Cl}: 0.25-1.00$ & $\mathrm{p}<0.05$ & 180 & & & Mathews 2006 [26] \\
\hline Maternal smoking (>10 cigarettes/d) & $\begin{array}{l}\text { TS vs. Control }(3 / 45 \text { vs. } 1 / 62, \mathrm{OR}=4.6,95 \% \\
\mathrm{Cl}=0.45-46.6, \mathrm{p}=0.19) ; \mathrm{TS}+\mathrm{ADHD} \text { vs. Control } \\
(7 / 60 \text { vs. } 1 / 62, \mathrm{OR}=8.5,95 \% \mathrm{Cl}=0.97-75.2 \\
p=0.052)\end{array}$ & $\begin{array}{l}p=0.19 \text { and } \\
0.05\end{array}$ & $\begin{array}{l}\text { TS: } 45, \text { TS + } \\
\text { ADHD:60 }\end{array}$ & 65 & $\begin{array}{l}\text { Adjusted for } \\
\text { gender }\end{array}$ & Motlagh 2010 [17] \\
\hline Maternal smoking & $\mathrm{TS}+\mathrm{ADHD}$ vs. TS, OR=2.43 (95\% Cl: $1.23-4.82)$ & $p=0.01$ & 181 & 172 & Adjusted OR & Pringsheim 2009 [21] \\
\hline Maternal smoking & Presence of OCD (OR=8.27, 95\% Cl=0.87-78.20) & $p=0.07$ & 180 & & & Mathews 2006 [26] \\
\hline Maternal alcohol exposure & TS + ADHD vs. TS, OR=0.81 (95\% Cl: 0.46-1.41) & $p=0.45$ & 181 & 172 & Adjusted OR & Pringsheim 2009 [21] \\
\hline \multirow[t]{2}{*}{ Coffee, cigarettes, alcohol } & Male TS vs. Female TS: $47 \%$ vs. $50 \%$ & NS & $46 \mathrm{M}$ & $7 \mathrm{~F}$ & & Santangelo 1994 [19] \\
\hline & TS + OCD vs. TS: OR=5.0, 95\% Cl: 1.3-19.4 & $\mathrm{p}<0.05$ & 15 & 34 & & Santangelo 1994 [19] \\
\hline \multicolumn{7}{|l|}{ Perinatal period } \\
\hline Disordered deliveries & $(16 / 42$ vs. $14 / 43)(\mathrm{OR}=1.27,95 \% \mathrm{Cl}=0.52-3.11)$ & $p=0.59$ & 42 & 43 & & Kondo 1982 [15] \\
\hline \multirow[t]{2}{*}{ Any delivery complication } & Male TS vs. Female TS: OR=9.4, 95\% Cl: 1.01-87.3 & $\mathrm{p}<0.05$ & $46 \mathrm{M}$ & $7 F$ & & Santangelo 1994 [19] \\
\hline & TS + OCD vs. TS: $75 \%$ vs. $54 \%$ & NS & 15 & 34 & & Santangelo 1994 [19] \\
\hline Operative delivery & TS + ADHD vs. TS, OR= 1.10 (95\% Cl: 0.71-1.71) & $p=0.66$ & 181 & 172 & Unadjusted OR & Pringsheim 2009 [21] \\
\hline \multirow[t]{2}{*}{ Forceps delivery } & Male TS vs. Female TS: $44 \%$ vs. $14 \%$ & NS & $46 \mathrm{M}$ & $7 \mathrm{~F}$ & & Santangelo 1994 [19] \\
\hline & TS + OCD vs. TS: OR=7.9, 95\% Cl: 3.2-19.5 & $\mathrm{p}<0.01$ & 15 & 34 & & Santangelo 1994 [19] \\
\hline Perinatal disorders: low BW, Asphyxia, severe & $8 / 43$ vs. $9 / 43, X \wedge 2=0.07(O R=0.86,95 \%$ & NS $(p=0.79)$ & 43 & 43 & & Kondo 1982 [15] \\
\hline
\end{tabular}


Table 2 Pre- and peri-natal factors associated with the onset of Tourette's syndrome (TS), and the presence of comorbidity (Continued)

\begin{tabular}{|c|c|c|c|c|c|c|}
\hline $\begin{array}{l}\text { Perinatal complications (postnatal jaundice, cord } \\
\text { around neck, breech birth, premature birth) }\end{array}$ & TS + OCS vs. TS only: 5/13 vs. 0/10) & $p<0.01$ & 13 & 10 & Fisher's exact test & lida 1996 [22] \\
\hline$>1$ hypoxic event & $\begin{array}{l}\text { TS vs. Control }(17 / 45 \text { vs. } 18 / 65, \mathrm{OR}=1.4,95 \% \\
\mathrm{Cl}=0.64-3.3, \mathrm{NS}) ; \mathrm{TS}+\mathrm{ADHD} \text { vs. Control } \\
\text { (18/60 vs. } 18 / 65, \mathrm{OR}=0.9,95 \% \mathrm{Cl}=0.4-2.2, \mathrm{NS})\end{array}$ & NS & $\begin{array}{l}\text { TS: } 45, \text { TS + } \\
\text { ADHD:60 }\end{array}$ & 65 & $\begin{array}{l}\text { Adjusted for } \\
\text { gender }\end{array}$ & Motlagh 2010 [17] \\
\hline Apgar 1 & $7.170+/-1.909$ vs. $7.517+/-1.623$ & $p=0.17$ & 53 & 265 & Matched analysis & Burd 1999 [11] \\
\hline Apgar 5 & $8.396+/-1.446$ vs.8.789+/-1.123 & $p=0.03$ & 53 & 265 & Matched analysis & Burd 1999 [11] \\
\hline Breathing problems at birth & TS + ADHD vs. TS, OR= 1.95 (95\% Cl: 0.87-4.37) & $p=0.10$ & 181 & 172 & Adjusted OR & Pringsheim 2009 [21] \\
\hline \multicolumn{7}{|l|}{ Combined pre- and peri-natal periods } \\
\hline $\begin{array}{l}\text { Total with abnormalities of prenatal and perinatal } \\
\text { periods }\end{array}$ & $19 / 51$ vs. $13 / 51(\mathrm{OR}=1.74,95 \% \mathrm{Cl}=0.74-4.05)$ & $p=0.20$ & 51 & 51 & & Pasamanick 1956 [18] \\
\hline $\begin{array}{l}\text { Reduced optimality in pre-, peri-, and neonatal } \\
\text { periods }\end{array}$ & $\begin{array}{l}\text { No statistical differences between cases and } \\
\text { controls (no data provided) in reduced optimality }\end{array}$ & & 25 & 25 & & Khalifa 2005 [13] \\
\hline Pre- and perinatal events & $\begin{array}{l}\text { TS }(26 / 48(54 \%)) \text {, Chronic tics }(24 / 48(50 \%)) \text { vs. } \\
\text { Control }(2 / 30(6 \%)) ; \text { TS: OR }=16.55(95 \% \\
C I=3.54-77.40) ; \text { Chronic tics: OR = } 14.00(95 \% \\
C I=3.00-65.44)\end{array}$ & $\begin{array}{l}\mathrm{p}<0.001 \\
\text { (both) }\end{array}$ & $\begin{array}{l}\text { TS: } 48 \text {, chronic } \\
\text { tics: } 48\end{array}$ & 30 & & Saccomani 2005 [23] \\
\hline \multicolumn{7}{|l|}{ Neonatal factors } \\
\hline Gestation age & 39.64 (2.73) vs. 40.04 (3.54) & 0.44 & 92 & 460 & Matched analysis & Burd 1999 [11] \\
\hline \multirow[t]{2}{*}{ Gestational age (<37 week) } & 5/106 vs. $62289 / 1153363$ & & 106 & 1153363 & & Shimada 2012 [20] \\
\hline & TS + ADHD vs. TS, OR= 3.83 (95\% Cl: 15.1-9.69) & $\mathrm{p}<0.01$ & 181 & 172 & Unadjusted OR & Pringsheim 2009 [21] \\
\hline Gestational age (<38 week) & TS: 1/45, TS + ADHD: 6/60, Control: 7/65 & NS & $\begin{array}{l}\text { TS: } 45, \text { TS }+ \\
\text { ADHD:60 }\end{array}$ & 65 & & Motlagh 2010 [17] \\
\hline Gestational age (>42 week) & TS: 4/45, TS + ADHD: 4/60, Control: 2/65 & NS & $\begin{array}{l}\text { TS: } 45, \text { TS }+ \\
\text { ADHD:60 }\end{array}$ & 65 & & Motlagh 2010 [17] \\
\hline \multirow[t]{3}{*}{ Birth Weight } & $3342.2+/-661.38$ vs. $3447.7+/-607.71$ & $p=0.14$ & 92 & 460 & Matched analysis & Burd 1999 [11] \\
\hline & $\begin{array}{l}\text { TS: } 3538+/-530, \text { TS + ADHD: } 3438+/-538, \\
\text { Control: } 3519+/-755\end{array}$ & NS & $\begin{array}{l}\text { TS: } 45, \text { TS }+ \\
\text { ADHD:60 }\end{array}$ & 65 & & Motlagh 2010 [17] \\
\hline & $\begin{array}{l}\text { TS vs. Disconcordant Twin: } 2228+-244 \text { vs. } \\
2545+-216\end{array}$ & $p=0.006$ & 6 & 6 & $\begin{array}{l}\text { Twins study } \\
\text { (paired t test) }\end{array}$ & Leckman 1987 [16] \\
\hline \multirow[t]{3}{*}{ Low birth weight (<2500 g) } & $\begin{array}{l}\text { TS vs. Control }(1 / 45 \text { vs. } 2 / 65, \mathrm{OR}=1.3,95 \% \\
\mathrm{Cl}=0.11-15.4, \mathrm{NS}) ; \mathrm{TS}+\mathrm{ADHD} \text { vs. Control } \\
(3 / 57 \text { vs. } 2 / 65, \mathrm{OR}=0.55,95 \% \\
\mathrm{Cl}=0.08-3.7, \mathrm{NS})\end{array}$ & NS & $\begin{array}{l}\text { TS: } 45, \text { TS }+ \\
\text { ADHD:60 }\end{array}$ & 65 & $\begin{array}{l}\text { adjusted for } \\
\text { gender }\end{array}$ & Motlagh 2010 [17] \\
\hline & TS vs. Control (1/116 vs. 9162/100097, OR=0.094) & & 116 & 100097 & & Shimada 2012 [20] \\
\hline & TS + ADHD vs. TS, OR=2.74 (95\% Cl: 1.03-7.29) & $p=0.04$ & 181 & 172 & Adjusted OR & Pringsheim 2009 [21] \\
\hline Small for gestational age ( $<10$ percentile) & TS + ADHD vs. TS, OR= $1.13(95 \% \mathrm{Cl}: 0.63-2.04)$ & $p=0.69$ & 181 & 172 & Unadjusted OR & Pringsheim 2009 [21] \\
\hline
\end{tabular}


Table 2 Pre- and peri-natal factors associated with the onset of Tourette's syndrome (TS), and the presence of comorbidity (Continued)

\begin{tabular}{lllll}
\hline $\begin{array}{l}\text { Birth weight above } 2500 \mathrm{~g} \\
\text { Birth order }\end{array}$ & Presence of ADHD, OR=0.69, 95\% Cl: 0.52-0.92 & $\mathbf{p}=\mathbf{0 . 0 2}$ & 180 & Mathews 2006 [26] \\
& $\mathrm{X} \wedge 2=2.53$ & NS & 43 & 43 \\
& First (78/116 vs. 53947/100118), second (34/116 vs. & 116 & 100118 \\
& $34594 / 100118),>=$ third (8/116 vs. 11577/100118) & Kondo 1982 [15] & Shimada 2012 [20]
\end{tabular}

First (78/116 vs. 53947/100118), second (34/116 vs,
$34594 / 100118),>=$ third (8/116 vs. 11577/100118)

*TS versus controls if not indicated. ADHD: Attention deficit hyperactive disorder; BW: birth weight; Cl: confidence interval; NS: non-significant; OCD: Obsessive compulsive disorder; OCS: Obsessive compulsive symptoms; OR: odds ratio; TS: Tourette's syndrome, statistically significant results shown in bold. 
prenatal period (adjusted OR $=2.43,95 \% \mathrm{CI} ; 1.23-4.82$, $\mathrm{p}=0.01$ ) [21]. Maternal smoking was also reported to be associated with a borderline higher risk for OCD $(\mathrm{OR}=8.27,95 \% \mathrm{CI}=0.87-78.20, \mathrm{p}=0.07)$ [26]. In another study combining coffee, cigarettes, and alcohol exposures as one single factor, the presence of comorbid OCD with TS was significantly associated with these exposures compared to TS only patients $(\mathrm{OR}=5.0,95 \%$ CI: 1.3-19.4, p < 0.05) [19].

In summary, although there were no consistent results among studies, many factors were reported to be significantly associated with the onset of TS or the presence of comorbidities in TS patients. These factors included earlier prenatal care, more prenatal visits, pregnancy complications, antibiotic exposure, maternal smoking, and coffee, cigarette, and alcohol exposure. Prenatal adversities during a critical developmental stage may be associated with the onset of TS or the development of comorbidities in these patients.

\section{Perinatal period}

The association between TS and perinatal risk factors was reported in 6 articles (Table 2) [11,15,17,19,21,22]. These studies suggest that while some perinatal risk factors were associated with specific conditions, the results are inconsistent. Among them, delivery complications were noted more frequently in boys with TS compared to girls $(\mathrm{OR}=9.4,95 \% \mathrm{CI}: 1.01-87.3, \mathrm{p}<$ 0.05) [19]. Forceps delivery was associated with the presence of OCD in TS patients compared to TS only $(\mathrm{OR}=7.9,95 \% \mathrm{CI}: 3.2-19.5, \mathrm{p}<0.01)$ [19]. In addition, Iida reported that perinatal complications were associated with the presence of obsessive-compulsive symptoms in TS patients compared to TS only [22]. For the onset of TS, Burd et al. (1999) reported that the Apgar score at 5 minutes was significantly lower in children with TS compared to controls $(8.396 \pm 1.446$ vs. $8.789 \pm$ 1.123, $\mathrm{p}=0.028$ ) [11]. Although significant associations between TS and the perinatal factors described above were reported in these 6 articles, many of these articles revealed non-significant results for different measurements. Compared to prenatal factors, a relatively smaller proportion of results for perinatal factors revealed significant associations with TS.

\section{Combined prenatal and perinatal period}

When prenatal and perinatal adversities were considered as one factor, only one of 3 studies revealed significant associations of prenatal and perinatal adversity with TS $(\mathrm{OR}=16.55,95 \% \mathrm{CI}=3.54-77.40, \mathrm{p}<0.001)$ or chronic tics $(\mathrm{OR}=14.0095 \% \mathrm{CI}=3.00-65.44, \mathrm{p}<0.001)$ (Table 2) [23]. However, in the other two studies, no associations were reported $[13,18]$.

\section{Neonatal factors}

The relationship between gestational age, birth weight, birth order and TS has been investigated in several studies (Table 2) [11,15-17,20,21]. Most studies reported nonsignificant results for these factors. In 4 articles investigating gestational age, only one study revealed that the presence of ADHD in TS patients was significantly associated with prematurity ( $<37$ weeks) compared to TS only patients in an unadjusted evaluation $(\mathrm{OR}=3.83,95 \% \mathrm{CI}$ : 15.1-9.69, $\mathrm{p}<0.01)$ [21]. The same study also found that low birth weight $(<2500 \mathrm{~g})$ was associated with a higher odds of comorbid TS and ADHD (OR $=2.74,95 \% \mathrm{CI}$ : 1.03-7.29, $\mathrm{p}=0.04$ ) [21]. Another study found that higher birth weight was associated with a lower risk of ADHD comorbidity in TS patients (OR $=0.69,95 \%$ CI: 0.52-0.92, $\mathrm{p}=0.02$ ) [26]. In a twin study where only one twin was affected by TS, lower birth weight was noted in the TS twin compared to their disconcordant twin without TS (TS twins: $2228 \pm 244$ gm vs. Twins without TS: $2545 \pm 216$ gm, paired $t$ test, $p=0.006$ ) [16]. For birth order, both studies found that it is not associated with the onset of TS $[15,20]$.

\section{Studies investigating the association between pre- and perinatal factors and severity of tic symptoms and comorbidities}

There were 5 articles investigating the association between pre- and perinatal factors and the severity of tic symptoms and comorbidities [24-28]. All articles included only TS patients using a single group cross sectional design with comparison of disease symptom severity between those exposed and not exposed to perinatal adversity. All of the significant and non-significant results in these articles were summarized in Table 3.

\section{Prenatal factors}

Some prenatal factors were reported to be significantly associated with the severity of tic symptoms or comorbidities (Table 3). Pregnancy complications which included hypertension, infections, (pre)eclampsia, psychosocial stress, or diabetes mellitus were reported to be associated with greater tic severity at a single time point as measured by the Yale Global Tic Severity Scale (YGTSS) [27]. However, these pregnancy complications were found to be associated with lower mean compulsive CYBOCS ratings in these patients [28]. In addition, severity of nausea/vomiting during pregnancy was also noted to be associated with current tic severity as measured using the TS Global Scale based on all available information (direct examination, review of videotapes, parental- and self-reports, school reports, and medical records) [24]. With respect to in utero medication exposure, this was associated with global tic severity and a borderline higher 
Table 3 Pre and perinatal factors associated with tic severity and severity of co-morbid ADHD, OCD, and other disorders

\begin{tabular}{|c|c|c|c|c|c|c|}
\hline Factors & Results (exposed vs. Non-exposed) & $P$ value & $\begin{array}{l}\text { Directions of } \\
\text { association }\end{array}$ & $\begin{array}{l}\text { Case } \\
\text { Number }\end{array}$ & $\begin{array}{l}\text { Scales of } \\
\text { measurements }\end{array}$ & Reference \\
\hline \multicolumn{7}{|l|}{ Pre-natal period } \\
\hline \multirow[t]{7}{*}{ Pregnancy complications } & Current YGTSS $22.3+/-9.4$ vs. $17.3+/-8.4$ & $p=0.03$ & $\uparrow$ & 70 & YGTSS & $\begin{array}{l}\text { Bos-Veneman } 2010 \\
{[27]}\end{array}$ \\
\hline & $2.0+-3.7$ vs. $3.9+-4.9$ & $p=0.05$ & $\downarrow$ & 110 & CYBOCS & $\begin{array}{l}\text { Bos-Veneman } 2010 \\
{[27]}\end{array}$ \\
\hline & No difference in worst ever tic severity & $p=0.68$ & - & 70 & YGTSS & $\begin{array}{l}\text { Bos-Veneman } 2010 \\
{[27]}\end{array}$ \\
\hline & No difference in ADHD severity & $p=0.45$ & - & 65 & ADHD rating scale & $\begin{array}{l}\text { Bos-Veneman } 2010 \\
{[27]}\end{array}$ \\
\hline & No difference in tic severity & NS & - & 110 & YGTSS & $\begin{array}{l}\text { Bos-Veneman } 2010 \\
{[27]}\end{array}$ \\
\hline & No difference in social behaviours & NS & - & 110 & CSBQ & $\begin{array}{l}\text { Bos-Veneman } 2010 \\
{[27]}\end{array}$ \\
\hline & No difference in anxiety and depression & NS & - & 110 & RCADS & $\begin{array}{l}\text { Bos-Veneman } 2010 \\
{[27]}\end{array}$ \\
\hline Severity of nausea/vomiting & Associated with current tic severity & $p=0.01$ & $\uparrow$ & 31 & $\begin{array}{l}\text { TS Global scale and } \\
\text { C-GAS (adjusted for } \\
\text { gender) }\end{array}$ & Leckman 1990 [24] \\
\hline \multirow[t]{2}{*}{ Number of medications exposed in utero } & Associated with increased global tic severity & $\mathrm{p}<0.00001$ & $\uparrow$ & 180 & YGTSS & Mathews 2006 [26] \\
\hline & Interference of OCS & $p=0.08$ & $\uparrow$ & 180 & & Mathews 2006 [26] \\
\hline \multirow[t]{10}{*}{ Maternal smoking } & ADHD Severity $26.4+-10.1$ vs. $19.1+-10.9$ & $p=0.05$ & $\uparrow$ & 70 & ADHD rating scale & $\begin{array}{l}\text { Bos-Veneman } 2010 \\
{[27]}\end{array}$ \\
\hline & $\begin{array}{l}\text { Associated with more severe ADHD } \\
\text { symptoms modified by positive family } \\
\text { history of mental disorders }\end{array}$ & $p=0.04$ & $\uparrow$ & 70 & ADHD rating scale & $\begin{array}{l}\text { Bos-Veneman } 2010 \\
{[27]}\end{array}$ \\
\hline & $9.1+-4.1$ vs. $6.1+-4.1$ & $p=0.05$ & $\uparrow$ & 110 & MDD RCADS & $\begin{array}{l}\text { Bos-Veneman } 2010 \\
{[27]}\end{array}$ \\
\hline & $6.6+-3.8$ vs. $4.4+-3.7$ & $p=0.05$ & $\uparrow$ & 110 & $\begin{array}{l}\text { CSBQ subscale } \\
\text { orientation ratings }\end{array}$ & $\begin{array}{l}\text { Bos-Veneman } 2010 \\
{[27]}\end{array}$ \\
\hline & Positive association with tic severity & $\mathrm{p}<0.00001$ & $\uparrow$ & 180 & YGTSS & Mathews 2006 [26] \\
\hline & Interference of OCS & $p=0.03$ & $\uparrow$ & 180 & & Mathews 2006 [26] \\
\hline & No difference in current tic severity & $p=0.38$ & - & 70 & YGTSS & $\begin{array}{l}\text { Bos-Veneman } 2010 \\
{[27]}\end{array}$ \\
\hline & No difference in worst ever tic severity & $p=0.86$ & - & 65 & YGTSS & $\begin{array}{l}\text { Bos-Veneman } 2010 \\
{[27]}\end{array}$ \\
\hline & No difference in tic severity & NS & - & 110 & YGTSS & $\begin{array}{l}\text { Bos-Veneman } 2010 \\
{[27]}\end{array}$ \\
\hline & $\begin{array}{l}\text { No difference in obsessive compulsive } \\
\text { severity }\end{array}$ & NS & - & 110 & CYBOCS & $\begin{array}{l}\text { Bos-Veneman } 2010 \\
{[27]}\end{array}$ \\
\hline
\end{tabular}


Table 3 Pre and perinatal factors associated with tic severity and severity of co-morbid ADHD, OCD, and other disorders (Continued)

\begin{tabular}{|c|c|c|c|c|c|c|}
\hline & $\begin{array}{l}\text { No difference in social behaviours other than } \\
\text { orientation ratings }\end{array}$ & NS & - & 110 & CSBQ & $\begin{array}{l}\text { Bos-Veneman } 2010 \\
{[27]}\end{array}$ \\
\hline & $\begin{array}{l}\text { No difference in anxiety and total scale of } \\
\text { RCADS }\end{array}$ & NS & - & 110 & RCADS & $\begin{array}{l}\text { Bos-Veneman } 2010 \\
{[27]}\end{array}$ \\
\hline \multirow[t]{3}{*}{ Maternal alcohol drinking } & No difference in current tic severity & $p=0.13$ & - & 70 & YGTSS & $\begin{array}{l}\text { Bos-Veneman } 2010 \\
{[27]}\end{array}$ \\
\hline & No difference in worst ever tic severity & $p=0.10$ & - & 70 & YGTSS & $\begin{array}{l}\text { Bos-Veneman } 2010 \\
{[27]}\end{array}$ \\
\hline & No difference in ADHD severity & $p=0.71$ & - & 65 & ADHD rating scale & $\begin{array}{l}\text { Bos-Veneman } 2010 \\
{[27]}\end{array}$ \\
\hline Psychological stress & Associated with current tic severity & $p=0.03$ & $\uparrow$ & 31 & $\begin{array}{l}\text { TS Global scale and } \\
\text { C-GAS }\end{array}$ & Leckman 1990 [24] \\
\hline \multicolumn{7}{|l|}{ Perinatal period } \\
\hline \multirow[t]{9}{*}{ Delivery complications } & Worst ever YGTSS $28.7+/-9.1$ vs. $23.4+/-7.3$ & $p=0.01$ & $\uparrow$ & 70 & YGTSS & $\begin{array}{l}\text { Bos-Veneman } 2010 \\
{[27]}\end{array}$ \\
\hline & $\begin{array}{l}\text { Associated with lesser total YGTSS ratings } \\
\text { after adjusting for age and gender modified } \\
\text { by DRD4 3R allele }\end{array}$ & $p=0.02$ & $\downarrow$ & 110 & YGTSS & $\begin{array}{l}\text { Bos-Veneman } 2010 \\
{[27]}\end{array}$ \\
\hline & $\begin{array}{l}\text { Associated with higher total anxiety ratings } \\
\text { and total RCADS after adjusting for age and } \\
\text { gender modified by DRD4 2R allele }\end{array}$ & $\begin{array}{l}p=0.004 \text { and } \\
0.006\end{array}$ & $\uparrow$ & 110 & RCADS & $\begin{array}{l}\text { Bos-Veneman } 2010 \\
{[27]}\end{array}$ \\
\hline & No difference in current tic severity & $p=0.82$ & - & 70 & YGTSS & $\begin{array}{l}\text { Bos-Veneman } 2010 \\
{[27]}\end{array}$ \\
\hline & No difference in ADHD severity & $P=0.13$ & - & 65 & ADHD rating scale & $\begin{array}{l}\text { Bos-Veneman } 2010 \\
{[27]}\end{array}$ \\
\hline & No difference in tic severity & NS & - & 110 & YGTSS & $\begin{array}{l}\text { Bos-Veneman } 2010 \\
{[27]}\end{array}$ \\
\hline & $\begin{array}{l}\text { No difference in obsessive compulsive } \\
\text { severity }\end{array}$ & NS & - & 110 & CYBOCS & $\begin{array}{l}\text { Bos-Veneman } 2010 \\
{[27]}\end{array}$ \\
\hline & No difference in social behaviours & NS & - & 110 & CSBQ & $\begin{array}{l}\text { Bos-Veneman } 2010 \\
{[27]}\end{array}$ \\
\hline & No difference in anxiety and depression & NS & - & 110 & RCADS & $\begin{array}{l}\text { Bos-Veneman } 2010 \\
{[27]}\end{array}$ \\
\hline $\begin{array}{l}\text { Delivery complications and other perinatal } \\
\text { adversities }\end{array}$ & $\begin{array}{l}\text { No difference in tic severity between TS } \\
\text { twins }\end{array}$ & NS & - & 13 pairs & Shapiro scales & Hyde 1992 [25] \\
\hline Severity of perinatal complications & Borderline associated with the tic severity & $p=0.07$ & $\uparrow$ & 31 & $\begin{array}{l}\text { TS Global scale and } \\
\text { C-GAS (adjusted for } \\
\text { gender) }\end{array}$ & Leckman 1990 [24] \\
\hline Number of perinatal complications & No association with the tic severity & NS & - & 31 & $\begin{array}{l}\text { TS Global scale and } \\
\text { C-GAS (adjusted for } \\
\text { gender) }\end{array}$ & Leckman 1990 [24] \\
\hline
\end{tabular}


Table 3 Pre and perinatal factors associated with tic severity and severity of co-morbid ADHD, OCD, and other disorders (Continued)

\section{Neonatal factors}

Birth Weight

Birth order in TS twins

Handedness in TS twins

\section{Postnatal period}

First-week postnatal complications
Higher tic scores in lighter body weight twin on both Shapiro and YGTSS scales

Within-pair body weight difference is correlated with within-pair tic score

difference $(r h o=0.72$ )

Mean first-born tic score vs. mean

second-born tic score: $7.1+-6.5$ vs.

$8.4+-5.6$

Tic score in right-handed vs. left-handed: $10.6+-4.7$ vs. $6.1+-4.6$

$\begin{array}{ll}\text { No difference in current tic severity } & p=0.87 \\ \text { No difference in worst ever tic severity } & p=0.74 \\ \text { No difference in ADHD severity } & p=0.83\end{array}$

$p=0.001$ and

$\mathrm{p}<0.005$

$p=0.57$

$p=0.13$

$p=0.83$
13 pairs

13 pairs

8 pairs

7 pairs

69
Shapiro scales and Hyde 1992 [25]

GISS

Shapiro scale Hyde 1992 [25]

Shapiro scales and Hyde 1992 [25] YGTSS

Shapiro scales and Hyde $1992[25]$ YGTSS

YGTSS

YGTSS

$[27]$

Bos-Veneman 2010

ADHD rating scale Bos-Veneman 2010

ADHD: Attention Deficit Hyperactive disorder; C-GAS: Children's Global Severity Scale; Cl: confidence interval; CSBQ: Children's Social Behavior Questionnaire; CYBOCS: Children's Yale-Brown Obsessive-Compulsive scale; DRD4: Dopamine receptor D4; MDD; Major Depression Disorder; NS: non-significant; OCD: Obsessive Compulsive Disorder; OCS: Obsessive-Compulsive Symptoms; OR: odds ratio; RCADS: Revised Child Anxiety and Depression Scale; TS Tourette's syndrome; YGTSS: Yale Global Tic Severity Scale; Borderline $(0.05<=p<0.10)$ or significant $(p<0.05)$ results are marked with the association directions, statistically significant results shown in bold. 
risk for obsessive compulsive symptoms but a lower risk for symptoms of ADHD in individuals with TS [26].

In addition to the prenatal factors mentioned above, some studies have investigated the association of tic severity or comorbidity severity with maternal smoking, maternal alcohol use, psychological stress during pregnancy, and the overall number of prenatal problems (Table 3). More severe ADHD rating scale scores, measured with the parent version of the ADHD Rating Scale, were associated with maternal smoking (smoking versus non-smoking: $26.4 \pm 10.1$ versus $19.1 \pm 10.9, \mathrm{p}=0.05)$ [27]. This association was more prominent when there was a positive family history of mental disorders $(\mathrm{p}=0.04)$ [27]. In another study, higher depression and autism scale scores, measured with the major depressive disorder subscale of the revised Child Anxiety and Depression Scale (RCADS) and the parent version of the Children's Social Behaviour Questionnaire (CSBQ), were noted in TS patients with a history of exposure to maternal smoking [28]. Maternal smoking was also reported to be associated with greater worst ever lifetime tic severity as measured by the YGTSS, and more obsessive compulsive symptoms in another study [26]. These results suggest an association between maternal smoking and tic, ADHD and OCD severity in individuals with TS. In addition to maternal smoking, maternal psychological stress was noted to be associated with greater tic severity [24]. With regard to the prenatal alcohol exposure, there was no association between maternal alcohol use and tic severity or ADHD comorbidity [27].

\section{Perinatal factors}

The presence of delivery complications including meconium-stained amniotic fluid, premature rupture of the membranes, nuchal cord, fetal bradycardia, placenta praevia, or artificial delivery was reported to be associated with higher worst ever tic severity (presence versus absence: $28.7 \pm 9.1$ vs. $23.4 \pm 7.3, \mathrm{p}=0.01$ ) as measured by the YGTSS [27].

Dopaminergic genes are candidate genes in TS studies given that alterations in dopaminergic neurotransmission are believed to contribute to the etiology of tics. In a study investigating the interaction between the dopamine receptor D4 (DRD4) gene and perinatal factors associated with tic severity, TS patients with the DRD4 3R allele and a history of delivery complications had a lower total tic scale rating, measured with the YGTSS, compared to individuals without the DRD4 3R allele $(\mathrm{p}=0.02)$ [28]. The same study also found that in patients with the DRD4 2R allele, higher total anxiety ratings and total Revised Child Anxiety and Depression Scale scores (RCADS) were noted in TS patients with delivery complications $(\mathrm{p}=0.004$ and 0.006 respectively). These results suggest an interaction of genetic and environmental factors on the severity of tics and associated comorbidities in TS patients.

\section{Neonatal factors}

In a twin study evaluating birth weight and lifetime tic severity, the twin with lower birth weight had higher tic scores measured with Shapiro Symptom Check List and YGTSS [25]. In the same study, the within-pair body weight difference was linearly correlated with the withinpair tic score difference measured with the Shapiro score $(\mathrm{rho}=0.72, \mathrm{p}<0.005)$ [25]. These findings suggested that lower birth weights were associated with the greater tic severity between identical twins. In addition, these associations had a linear dose-response relationship. These results suggest that lower birth weight is associated with higher tic scores in TS patients.

\section{Time trends and seasonal variation}

There was one article investigating time trends in the incidence of Tourette syndrome in a population-based cohort [30]. In this study, a statistically significant increase was found in the cumulative incidence across specific birth years for TS from 1990 to 1995. For example, for children age 9, the cumulative incidences are 2.3 (95\% CI: 1.4-3.1), 2.4 (95\% CI: 1.5-3.2), and 3.9 (95\% CI: 2.94.9) for birth cohort in 1990-1991, 1992-1993, and 1994-1995, respectively. The study could not identify if there were environmental factors including pre- or perinatal adversities associated with this increase in incidence, or whether this was due to an improvement in identification and diagnosis of tic disorders over this time period. Another study was performed using the same cohort data to evaluate the seasonal variation in the incidence of TS. The results found that there was no evidence of seasonal variation of birth and TS incidence [29].

\section{Other types of studies}

Some indirect evidence also suggests that perinatal difficulties may play a role in the onset of TS. In one study investigating TS probands and relatives' risks for TS, perinatal difficulties in TS probands were inversely related to the recurrence risk among first-degree relatives of TS probands [36]. This suggests that TS probands with perinatal problems had a lower risk of TS in first degree relatives. It provides indirect evidence that part of the risk for TS in these probands was related to these perinatal insults. In another study investigating the association between neonatal cranial ultrasound abnormalities and the prevalence of tic disorders in low-birth-weight children, the prevalence of tic disorders at age of 6 was significantly associated with parenchymal lesions and/or ventricular enlargement in neonatal cranial ultrasound examinations $(\mathrm{OR}=8.7,95 \% \mathrm{CI}: 1.3-57.7, \mathrm{p}=0.02)$ [39]. These results 
suggest that neonatal brain injury might increase the risk for the onset of tic disorders.

\section{Discussion}

Overall, there were few articles investigating the association between pre- and perinatal adversities and the onset and severity of TS, and the presence and severity of comorbid disorders. Although some studies have reported associations between pre- and perinatal factors and TS, many of the results are inconsistent. Taken as a whole, there is only very weak evidence to support that pre- and perinatal adversity are associated with the development of TS, TS symptom severity and the presence or severity of comorbidities.

One of the major issues regarding the available literature on the relationship between TS and pre and perinatal risk factors is the overall poor methodological quality of the studies performed to date. Only five studies evaluated data on perinatal events that was collected prospectively on registered birth certificates or as part of a birth registry. The majority of studies used clinically ascertained samples of individuals with TS, which may differ substantially in disease severity than those ascertained through population based means. The available data are therefore susceptible to bias.

In comparing the results of the case control studies using birth certificate data with those who ascertained information about perinatal events from mothers retrospectively, there is no clear difference in the strength of association between TS and various perinatal risk factors. Burd [11] reported significantly earlier prenatal care, more prenatal visits, a lower odds of having care begun after the first trimester (three highly related factors), and lower Apgar scores at 5 minutes among cases than controls. Pasamanick [18] did not detect any significant relationships between abnormalities during the pre or perinatal period and TS.

Of the five studies examining the relationship between perinatal adversity and TS and comorbidity symptom severity, the majority of positive results reported would not remain significant after appropriate statistical correction for multiple hypothesis testing. The exception is the finding reported by Matthews [26] of the association between increased global tic severity and the number of medications reported in utero and maternal smoking (both $\mathrm{p}<0.00001$ ).

Of all the risk factors studied, maternal smoking and low birth weight appear to be the only risk factor with consistent significant associations. Maternal smoking was reported to be associated with the presence of comorbid ADHD and OCD, and TS, ADHD and OCD symptom severity. Although these results suggest a connection between maternal smoking and the onset of TS, other factors linking these exposures to TS need to be considered.
Nicotine has a therapeutic effect for some TS and ADHD patients [41-46]. Nicotine has been shown to reduce complex tics and improve behaviours related to inattention in TS patients [41]. Individuals with ADHD have a higher risk of smoking and nicotine dependence from adolescence to young adulthood [47]. If mothers with tics or ADHD smoke, the association of maternal smoking and TS in the offspring might be due to heritability of these disorders rather than smoking itself. Many individuals are unaware of their tics and are never diagnosed with TS and therefore may deny a family history of the disorder. This confounding effect should be carefully examined in future studies to clarify the association of prenatal smoking exposure and the onset or severity of TS, and the presence and severity of comorbid ADHD and OCD.

Most studies did not report significant associated neonatal factors with TS, except for low birth weight. Low birth weight was found to be associated with comorbid ADHD and tic severity. Low birth weight may be an independent factor associated with TS or comorbidities, or may be a consequence of prenatal adversity. Although this effect would be minimized in twin studies, further investigations are necessary to identify whether low birth weight itself is associated with TS or if it is the proxy of other prenatal insults.

The limitations of this systematic review include the overall poor methodological quality of the included studies, with differing study designs and analytic methods, and extreme diversity of measurements among different studies. Definitions of prenatal or perinatal adversities differed between studies, and were most commonly retrospectively ascertained. Finally, as the clinical symptoms of TS wax and wane during the disease course, the measurement of tic severity at a single point of time does not truly represent disease severity for patients. Although it is difficult to draw a valid conclusion from this literature, many preand perinatal adversities were reported to be associated with the onset of TS, the presence of comorbidities, and the severity of tics and comorbidities. Future studies are likely to make a significant contribution, especially if they include population derived cohorts, use data on perinatal events that was obtained prospectively, and use methods to control for multiple hypothesis testing. Standards for defining pre and perinatal events should be adhered to, and TS symptom severity should be measured at multiple time points if this outcome is being considered.

The mechanism through which prenatal and perinatal events lead to TS onset or worsening tic severity is unknown. These factors could contribute to microscopic abnormalities in brain structure or function that lead to the development of tics. TS is a neurodevelopmental disorder which shares common features with autism and attention deficit hyperactivity disorder. Dysfunction of the dopaminergic system has been implicated in all three 
disorders, and evidence from animal studies suggest that prenatal stress may cause changes in the dopaminergic system [48-51]. The frequent comorbidity of these neurodevelopmental disorders in clinical practice suggests a common pathophysiological basis, with shared prenatal and perinatal risk factors in autism and ADHD supporting these relationships [52-54].

\section{Conclusions}

In conclusion, this systematic review provides weak evidence for an association between pre- and perinatal adversity and the onset of TS, comorbid disorders, and the severity of tics, ADHD and OCD symptoms. Of all the risk factors studied, maternal smoking and low birth weight appear to be most consistently implicated. Studies of higher methodological quality are needed in order to better understand the relationship between prenatal and perinatal events and TS.

\section{Additional file}

Additional file 1: Medline search strategy.

\section{Competing interests}

Ting-Kuang Chao, Jing Hu and Tamara Pringsheim declare that they have no competing interests.

\section{Authors' contributions}

TKC performed the data extraction and synthesis, wrote the initial draft of the manuscript, and made subsequent revisions to the manuscript. JH created and executed the search strategy for original research articles, reviewed all abstracts for inclusion criteria, and assisted with revision of the manuscript. TP obtained grant funding for the study, created the search strategy for original research articles, reviewed all abstracts for inclusion criteria, and revised the manuscript. All authors read and approved the final manuscript.

\section{Acknowledgements}

This study is part of the National Population Health Study of Neurological Conditions. We wish to acknowledge the membership of Neurological Health Charities Canada and the Public Health Agency of Canada for their contribution to the success of this initiative.

Funding for the study was provided by the Public Health Agency of Canada. The opinions expressed in this publication are those of the authors/ researchers, and do not necessarily reflect the official views of the Public Health Agency of Canada.

\section{Author details}

${ }^{1}$ Department of Medicine, University of Alberta, Alberta, Canada. ${ }^{2}$ Department of Clinical Neurosciences, University of Calgary, Calgary, Canada. ${ }^{3}$ Alberta Children's Hospital, C4-431, 2888 Shaganappi Trail NW, Calgary, AB T3B 6A8, Canada.

Received: 23 May 2013 Accepted: 14 January 2014 Published: 30 January 2014

\section{References}

1. Bloch $M$, State $M$, Pittenger C: Recent advances in Tourette syndrome. Curr Opin Neurol 2011, 24(2):119-25.

2. Abelson JF, et al: Sequence variants in SLITRK1 are associated with Tourette's syndrome. Science 2005, 310(5746):317-20.

3. Ercan-Sencicek AG, et al: L-histidine decarboxylase and Tourette's syndrome. N Engl J Med 2010, 362(20):1901-8.
4. Verkerk AJ, et al: CNTNAP2 is disrupted in a family with Gilles de la Tourette syndrome and obsessive compulsive disorder. Genomics 2003, 82(1):1-9.

5. Chou IC, et al: Association of the Slit and Trk-like 1 gene in Taiwanese patients with Tourette syndrome. Pediatr Neurol 2007, 37(6):404-6.

6. Deng $\mathrm{H}$, et al: Examination of the SLITRK1 gene in Caucasian patients with Tourette syndrome. Acta Neurol Scand 2006, 114(6):400-2.

7. Fabbrini $\mathrm{G}$, et al: A large Italian family with Gilles de la Tourette syndrome: clinical study and analysis of the SLITRK1 gene. Mov Disord 2007, 22(15):2229-34.

8. Orth $\mathrm{M}$, et al: Autosomal dominant myoclonus-dystonia and Tourette syndrome in a family without linkage to the SGCE gene. Mov Disord 2007, 22(14):2090-6.

9. Zimprich A, et al: Sequence analysis of the complete SLITRK1 gene in Austrian patients with Tourette's disorder. Psychiatr Genet 2008, 18(6):308-9.

10. Alexander GM, Peterson BS: Testing the prenatal hormone hypothesis of tic-related disorders: gender identity and gender role behavior. Dev Psychopathol 2004, 16(2):407-20.

11. Burd $L$, et al: Prenatal and perinatal risk factors for Tourette disorder. J Perinat Med 1999, 27(4):295-302.

12. Csabi G, et al: Minor physical anomalies in Tourette syndrome. Eur J Psychiatry 2008, 22(3):173-180.

13. Khalifa N, Von Knorring AL: Tourette syndrome and other tic disorders in a total population of children: Clinical assessment and background. Acta Paediatr, Int J Paediatr 2005, 94(11):1608-1614.

14. Klug MG, et al: A comparison of the effects of parental risk markers on pre- and perinatal variables in multiple patient cohorts with fetal alcohol syndrome, autism, Tourette syndrome, and sudden infant death syndrome: an enviromic analysis. Neurotoxicol Teratol 2003, 25(6):707-17.

15. Kondo K, Nomura Y: Tourette syndrome in Japan: etiologic considerations based on associated factors and familial clustering. Adv Neurol 1982, 35:271-6.

16. Leckman JF, et al: Nongenetic factors in Gilles de la Tourette's syndrome. Arch Gen Psychiatry 1987, 44(1):100.

17. Motlagh $\mathrm{MG}$, et al: Severe psychosocial stress and heavy cigarette smoking during pregnancy: an examination of the pre- and perinatal risk factors associated with ADHD and Tourette syndrome. Eur Child Adolesc Psychiatry 2010, 19(10):755-764.

18. Pasamanick B, Kawi A: A study of the association of prenatal and paranatal factors with the development of tics in children; a preliminary investigation. J Pediatr 1956, 48(5):596-601.

19. Santangelo SL, et al: Tourette's syndrome: what are the influences of gender and comorbid obsessive-compulsive disorder? J of the Am Academy of Child \& Adolesc Psychiatry 1994, 33(6):795-804.

20. Shimada T, et al: Parental age and assisted reproductive technology in autism spectrum disorders, attention deficit hyperactivity disorder, and Tourette syndrome in a Japanese population. Res Autism Spectrum Disord 2012, 6(1):500-507.

21. Pringsheim $T$, et al: Prenatal and perinatal morbidity in children with Tourette syndrome and attention-deficit hyperactivity disorder. J Dev Behav Pediatr 2009, 30(2):115-21.

22. lida J, et al: The clinical features of Tourette's disorder with obsessivecompulsive symptoms. Psychiatry Clin Neurosci 1996, 50(4):185-9.

23. Saccomani $L$, et al: Tourette syndrome and chronic tics in a sample of children and adolescents. Brain Dev 2005, 27(5):349-52.

24. Leckman JF, et al: Perinatal factors in the expression of Tourette's syndrome: An exploratory study. J Am Acad Child Adolesc Psychiatry 1990, 29(2):220-226.

25. Hyde TM, et al: Relationship of birth weight to the phenotypic expression of Gilles de la Tourette's syndrome in monozygotic twins. Neurology 1992, 42(3 Pt 1):652-8.

26. Mathews CA, et al: Association between maternal smoking and increased symptom severity in Tourette's syndrome. Am J Psychiatr 2006, 163(6):1066-73.

27. Bos-Veneman NG, et al: Role of perinatal adversities on tic severity and symptoms of attention deficit/hyperactivity disorder in children and adolescents with a tic disorder. J Dev Behav Pediatr 2010, 31(2):100-106.

28. Bos-Veneman NGP, Minderaa RB, Hoekstra PJ: The DRD4 gene and severity of tics and comorbid symptoms: main effects and interactions with delivery complications. Mov Disord 2010, 25(10):1470-6. 
29. Atladottir $\mathrm{HO}$, et al: Variation in incidence of neurodevelopmental disorders with season of birth. Epidemiology 2007, 18(2):240-5.

30. Atladottir $\mathrm{HO}$, et al: Time trends in reported diagnoses of childhood neuropsychiatric disorders: a Danish cohort study. Arch Pediatr Adolesc Med 2007, 161(2):193-8.

31. Eapen $V$, et al: Clinical features and associated psychopathology in a Tourette syndrome cohort. Acta Neurol Scand 2004, 109(4):255-60.

32. Fernandez-Mayoralas DM, et al: Fetal alcohol syndrome, Tourette syndrome, and hyperactivity in nine adopted children. Pediatr Neurol 2010, 43(2):110-6.

33. Griffiths BJ, Hafez A, Stern JS: Month of birth of people attending a tourette syndrome clinic. J Neurol Neurosurg Psychiatry 2010, 81(10):e14.

34. Hyde TM, et al: Electroencephalographic abnormalities in monozygotic twins with Tourette's syndrome. Br J Psychiatry 1994, 164(6):811-817.

35. Incagnoli T, Kane R: Developmental perspective of the Gilles de la Tourette syndrome. Percept Mot Skills 1983, 57(3 Pt 2):1271-81.

36. Kano Y, Leckman JF, Pauls DL: Clinical characteristics of Tourette syndrome probands and relatives' risk. J Am Acad Child Adolesc Psychiatry 2002, 41(10):1148-1149.

37. Micheli F, et al: Gilles de la Tourette syndrome: Clinical features of 75 cases from Argentina. Behav Neurol 1995, 8(2):75-80.

38. Shapiro AK, Shapiro E, Wayne H: Birth, developmental, and family histories and demographic information in Tourette's syndrome. J Nerv Ment Dis 1972, 155(5):335-44.

39. Whitaker $\mathrm{AH}$, et al: Psychiatric outcomes in low-birth-weight children at age 6 years: relation to neonatal cranial ultrasound abnormalities. Arch Gen Psychiatry 1997, 54(9):847-56.

40. Zelnik N, et al: Height distribution in children with Tourette syndrome. J Child Neurol 2002, 17(3):200-4.

41. Howson $A L$, et al: Clinical and attentional effects of acute nicotine treatment in Tourette's syndrome. Eur Psychiatry 2004, 19(2):102-12.

42. Levin ED, et al: Nicotine effects on adults with attention-deficit/ hyperactivity disorder. Psychopharmacology (Berl) 1996, 123(1):55-63.

43. McConville BJ, et al: The effects of nicotine plus haloperidol compared to nicotine only and placebo nicotine only in reducing tic severity and frequency in Tourette's disorder. Biol Psychiatry 1992, 31(8):832-40.

44. Potter AS, Newhouse PA: Acute nicotine improves cognitive deficits in young adults with attention-deficit/hyperactivity disorder. Pharmacol Biochem Behav 2008, 88(4):407-17.

45. Silver $A A$, et al: Case study: long-term potentiation of neuroleptics with transdermal nicotine in Tourette's syndrome. J Am Acad Child Adolesc Psychiatry 1996, 35(12):1631-6.

46. Silver AA, et al: Transdermal nicotine and haloperidol in Tourette's disorder: a double-blind placebo-controlled study. J Clin Psychiatry 2001, 62(9):707-14

47. Fuemmeler BF, Kollins SH, McClernon FJ: Attention deficit hyperactivity disorder symptoms predict nicotine dependence and progression to regular smoking from adolescence to young adulthood. J Pediatr Psychol 2007, 32(10):1203-13.

48. Baier $C J$, et al: Gestational restraint stress and the developing dopaminergic system: an overview. Neurotox Res 2012, 22(1):16-32.

49. Makkonen I, et al: Effects of fluoxetine treatment on striatal dopamine transporter binding and cerebrospinal fluid insulin-like growth factor-1 in children with autism. Neuropediatrics 2011, 42(5):207-9.

50. Rogers TD, et al: Reorganization of circuits underlying cerebellar modulation of prefrontal cortical dopamine in mouse models of autism spectrum disorder. Cerebellum 2013, 12(4):547-556.

51. Staal WG, de Krom M, de Jonge MV: Brief report: the dopamine-3-receptor gene (DRD3) is associated with specific repetitive behavior in autism spectrum disorder (ASD). J Autism Dev Disord 2012, 42(5):885-8.

52. Gardener $H$, Spiegelman D, Buka SL: Perinatal and neonatal risk factors for autism: a comprehensive meta-analysis. Pediatrics 2011, 128(2):344-55.

53. Guinchat $V$, et al: Pre-, peri- and neonatal risk factors for autism. Acta Obstet Gynecol Scand 2012, 91(3):287-300.

54. Latimer $K$, et al: Disruptive behaviour disorders: a systematic review of environmental antenatal and early years risk factors. Child Care Health Dev 2012, 38(5):611-28.

\section{Submit your next manuscript to BioMed Central and take full advantage of:}

- Convenient online submission

- Thorough peer review

- No space constraints or color figure charges

- Immediate publication on acceptance

- Inclusion in PubMed, CAS, Scopus and Google Scholar

- Research which is freely available for redistribution 